\title{
Recombinant human erythropoietin attenuates hepatic dysfunction by suppressing hepatocellular apoptosis in lipopolysaccharide-induced disseminated intravascular coagulation in rats
}

\author{
YUKIO SUGA $^{1}$, FUMIO AKITA ${ }^{1}$, SHINYA YAMADA ${ }^{2}$, ERIKO MORISHITA $^{2}$ and HIDESAKU ASAKURA ${ }^{2}$ \\ ${ }^{1}$ Department of Clinical Pharmacy and Healthcare Science, Faculty of Pharmacy, \\ Institute of Medical, Pharmaceutical and Health Science, Kanazawa University; \\ ${ }^{2}$ Department of Hematology, Kanazawa University Hospital, Takaramachi, \\ Kanazawa, Ishikawa 920-8641, Japan
}

Received August 24, 2021; Accepted October 25, 2021

DOI: $10.3892 /$ br.2021.1488

\begin{abstract}
The aim of the present study was to clarify the effect of recombinant human erythropoietin (EPO) and low molecular weight heparin (LMWH) on a rat model of lipopolysaccharide (LPS)-induced disseminated intravascular coagulation (DIC). Experimental DIC was induced by sustained infusion of $5 \mathrm{mg} / \mathrm{kg}$ LPS for $4 \mathrm{~h}$. EPO or LMWH was then administered to the LPS-induced DIC model. LPS-induced consumption coagulopathy, hemostatic activation and plasma TNF elevation remained unaltered in the LPS+EPO group, except for the D-dimer levels, and these abnormalities were significantly improved in the LPS+LMWH group. Plasma alanine aminotransferase (ALT) levels were markedly reduced in the LPS+EPO group, accompanied by a significant suppression of hepatocellular apoptosis. In the LPS+LMWH group, plasma creatinine levels and glomerular fibrin deposition were significantly attenuated, along with plasma ALT levels and hepatocellular apoptosis. Thus, a single administration of EPO may improve hepatic dysfunction by primarily exerting an anti-apoptotic, not anticoagulant, effect in the LPS-induced DIC model.
\end{abstract}

\section{Introduction}

Disseminated intravascular coagulation (DIC) is a serious condition in which coagulation and fibrinolysis in the

Correspondence to: Dr Yukio Suga, Department of Clinical Pharmacy and Healthcare Science, Faculty of Pharmacy, Institute of Medical, Pharmaceutical and Health Science, Kanazawa University, 13-1 Takaramachi, Kanazawa, Ishikawa 920-8641, Japan

E-mail: suga@staff.kanazawa-u.ac.jp

Key words: disseminated intravascular coagulation, sepsis, erythropoietin, apoptosis, hepatic dysfunction circulating blood are markedly increased. The characteristic clinical manifestations of DIC are organ failure and an increased tendency of bleeding. DIC can result in multi-organ failure (MOF) due to multiple microthrombi in the microvasculature of multiple organs $(1,2)$.

Lipopolysaccharide (LPS) is frequently used to induce DIC in experimental animal models. The pathophysiology of LPS-induced DIC is well documented, and mimics the type of DIC observed in patients with sepsis $(3,4)$. The profibrinolytic response is almost immediately followed by the suppression of fibrinolytic activity. This suppression is induced by a sustained increase in the plasma levels of plasminogen activator inhibitor in the LPS-induced DIC model $(5,6)$. The activation of coagulation and impairment of fibrinolysis has been shown to be mediated by cytokines, such as TNF, IL-1 and IL-6 in the LPS-induced DIC model $(7,8)$.

Erythropoietin (EPO) is a 30,400-dalton glycoprotein that regulates red cell production. In humans, EPO is produced by peritubular cells in the kidneys, and causes maturation and proliferation of erythroid progenitor cells (9). Recombinant human EPO is licensed worldwide for the treatment of anemia in patients with chronic renal failure $(10,11)$. EPO protects several organs, including the brain, heart, kidneys and liver against injury caused by ischemia/reperfusion and excessive inflammation, and these beneficial effects of EPO are associated with reductions in tissue apoptosis secondary to prevention of the activation of caspase-3,-8 and -9 (12-14). The tissue-protective effects of EPO following its anti-apoptotic properties have also been reported in rodent models of sepsis, using human recombinant EPO and its analogue $(15,16)$. Numerous studies have shown that endothelial cells can undergo apoptosis in response to polymicrobial endotoxic shock $(17,18)$. Endothelial apoptosis enhances exposure of negatively charged phospholipids, which are involved in factor VIIIa- and factor IXa-dependent activation of factor $\mathrm{X}$ on endothelial cells $(19,20)$. Encouraged by these studies, it was hypothesized that EPO may abrogate LPS-induced DIC with MOF. 
The aim of the present study was to clarify the role of apoptosis in LPS-induced DIC by investigating the effects of EPO. Specifically, whether EPO attenuated organ dysfunction and apoptosis in the LPS-induced rat DIC model was investigated. The effects of EPO on LPS-induced DIC were also compared with that of low molecular weight heparin (LMWH). This is the first report to investigate the effects of EPO on an animal model of DIC, to the best of our knowledge.

\section{Materials and methods}

Animals. Animals were maintained according to the Standards of Animal Care and Experimentation published by Kanazawa University. All animal experiments were approved by the Committee on Animal Experimentation at Kanazawa University (Kanazawa, Japan; approval no. AP-183920). Male Wistar rats (aged, 6-7 weeks; body weight, 160-170 g; Nippon SLC) were acclimatized for at least 3 days in the animal quarters before experimentation, and maintained with a 12-h light/dark cycle (lights on from between 8:45 a.m. and $8: 45$ p.m.) at a temperature of $24-26^{\circ} \mathrm{C}$ and humidity of 45-65\%, with free access to water and a regular diet (CRF-1; Charles River Laboratories, Inc.). A total of 126 rats were used in the present study.

Experimental procedure. Rats were anesthetized with isoflurane $(3 \%, 0.3 \mathrm{l} / \mathrm{min}$, inhaled). Blood was withdrawn from the abdominal aorta into plastic syringes at 4,8 and $12 \mathrm{~h}$ after the start of LPS administration. All samples were diluted (1:9 v/v) with $4 \%$ sodium citrate. Rats were sacrificed by death from exsanguination due to blood sampling from the abdominal aorta under deep anesthesia with isoflurane $(3 \%, 0.3 \mathrm{l} / \mathrm{min}$, inhaled).

The control groups were administered a sustained infusion of $10 \mathrm{ml}$ physiological saline for $4 \mathrm{~h}$ via the tail vein in the first experiment. Blood was withdrawn 4,8 and $12 \mathrm{~h}$ after this infusion ( $\mathrm{n}=7$ for each control group).

LPS (Escherichia coli 055: B5 lipopolysaccharide; Sigma-Aldrich; Merck KGaA) was freshly dissolved in physiological saline before each experiment. Experimental DIC was induced by sustained infusion of $5 \mathrm{mg} / \mathrm{kg}$ LPS diluted in $10 \mathrm{ml}$ saline into the tail vein over $4 \mathrm{~h}(\mathrm{n}=7)$ (LPS group). Blood was withdrawn 4,8 and $12 \mathrm{~h}$ after the start of LPS administration.

EPO (Epoetin $\alpha, 10,000$ IU/kg; Kyowa Hakko Kirin Co.) was administered to rats from $0.5 \mathrm{~h}$ before LPS infusion until LPS infusion was started ( $\mathrm{n}=7$ per group) (LPS+EPO groups). EPO diluted in $1.25 \mathrm{ml}$ saline was infused over the first $0.5 \mathrm{~h}$, after which LPS diluted in $10 \mathrm{ml}$ saline was infused over the next $4 \mathrm{~h}$. Blood was withdrawn 4,8 and $12 \mathrm{~h}$ after the start of LPS administration. The above administration schedule of EPO was used as pretreatment as it was the primary form of EPO administration used in previous studies investigating the effects of EPO on animal models of sepsis $(15,16)$, and because plasma concentrations of EPO were reportedly highly maintained $12 \mathrm{~h}$ after the intravenous administration of EPO in healthy rats (21). In the EPO-only groups (without LPS), $1.25 \mathrm{ml}$ physiological saline with EPO was infused over the first $0.5 \mathrm{~h}$, after which $10 \mathrm{ml}$ physiological saline was infused over the next $4 \mathrm{~h}$ via the tail vein. Blood was withdrawn 4,8 and $12 \mathrm{~h}$ after the start of physiological saline.
Infusion of LMWH (400 IU/kg) (Kissei Pharmaceutical Co.) was started from $0.5 \mathrm{~h}$ prior to LPS infusion. LMWH was administered for $4.5 \mathrm{~h}$ until LPS infusion was finished ( $n=7$ per group) (LPS+LMWH group). LMWH diluted in $1.25 \mathrm{ml}$ saline was infused over the first $0.5 \mathrm{~h}$, after which LPS and LMWH diluted in $10 \mathrm{ml}$ saline were simultaneously infused over the next $4 \mathrm{~h}$. Blood was withdrawn 4, 8 and $12 \mathrm{~h}$ after starting LPS administration. The above administration schedule of LMWH was used as plasma levels of D-dimer, fibrinogen, and thrombin-antithrombin complex were significantly suppressed by this administration schedule of LMWH in previous investigations on LPS-induced DIC in rats $(22,23)$. In the LMWH groups (without LPS), $1.25 \mathrm{ml}$ physiological saline with LMWH were simultaneously infused over the first $0.5 \mathrm{~h}$, after which $10 \mathrm{ml}$ physiological saline with LMWH was infused over the next $4 \mathrm{~h}$ via the tail vein. Blood was withdrawn 4, 8 and $12 \mathrm{~h}$ after the start of physiological saline administration.

Parameters. Platelets were counted using an automated device for animals (cat. no. MEK-6558; Celltac $\alpha$; Nihon Kohden Co.) within $1 \mathrm{~h}$ of sampling. Citrated plasma samples obtained by whole-blood centrifugation were stored at $-80^{\circ} \mathrm{C}$ until required. Fibrinogen concentration and prothrombin time (PT) were determined using a clotting assays according to the manufacturer's protocol (Fibrinogen determination and Thromborel S; Sysmex Co.). D-dimer levels were determined using a quantitative latex agglutination test according to the manufacturer's protocol (ELPIA ACE DD dimer; LSI Medience). Plasma levels of TNF were measured using a rat ELISA kit (cat. no. MBS2507393; BioSource). To determine the extent of organ dysfunction in rats, the plasma levels of creatinine and alanine aminotransferase (ALT) were determined using enzymatic (PureautoS CRE-L; cat. no. 20800AMZ10178000; Sekisui Medical Co.) and ultraviolet (Ltypewako ALT J2; FUJIFILM Wako Pure Chemical Corporation) determinations according to the manufacturer's protocol, respectively.

Pathological examination. Renal tissue specimens were obtained from animals sacrificed immediately after blood sampling, only at $8 \mathrm{~h}$ after starting LPS or saline infusion, as in our previous study it was shown that fibrin deposition in glomeruli could be assessed $8 \mathrm{~h}$ after starting LPS administration (22), and then fixed in formalin. The ratio (as a percentage) of glomerular fibrin deposition (GFD) was determined by microscopy. After staining specimens with phosphotungstic acid hematoxylin (12-24 h), each sample was histologically examined by a pathologist who was blinded to sample group allocations. A total of 100 glomeruli were examined in each sample, and the numbers of thrombi containing fibrin was expressed as a percentage.

Hepatic tissue specimens were fixed in formalin. The apoptotic index (AI) was determined by microscopy. After staining of hepatic tissue specimens using a TUNEL assay according to the manufacturer's protocol (in situ Apoptosis Detection kit; Takara Bio Inc.), each sample was histologically examined by a pathologist blinded to the sample groups. Apoptotic cells were countered in 10 random fields of view using a light microscope (magnification, x200) (24). The AI per sample was calculated as follows: $\mathrm{AI}=$ (number of apoptotic cells/total 

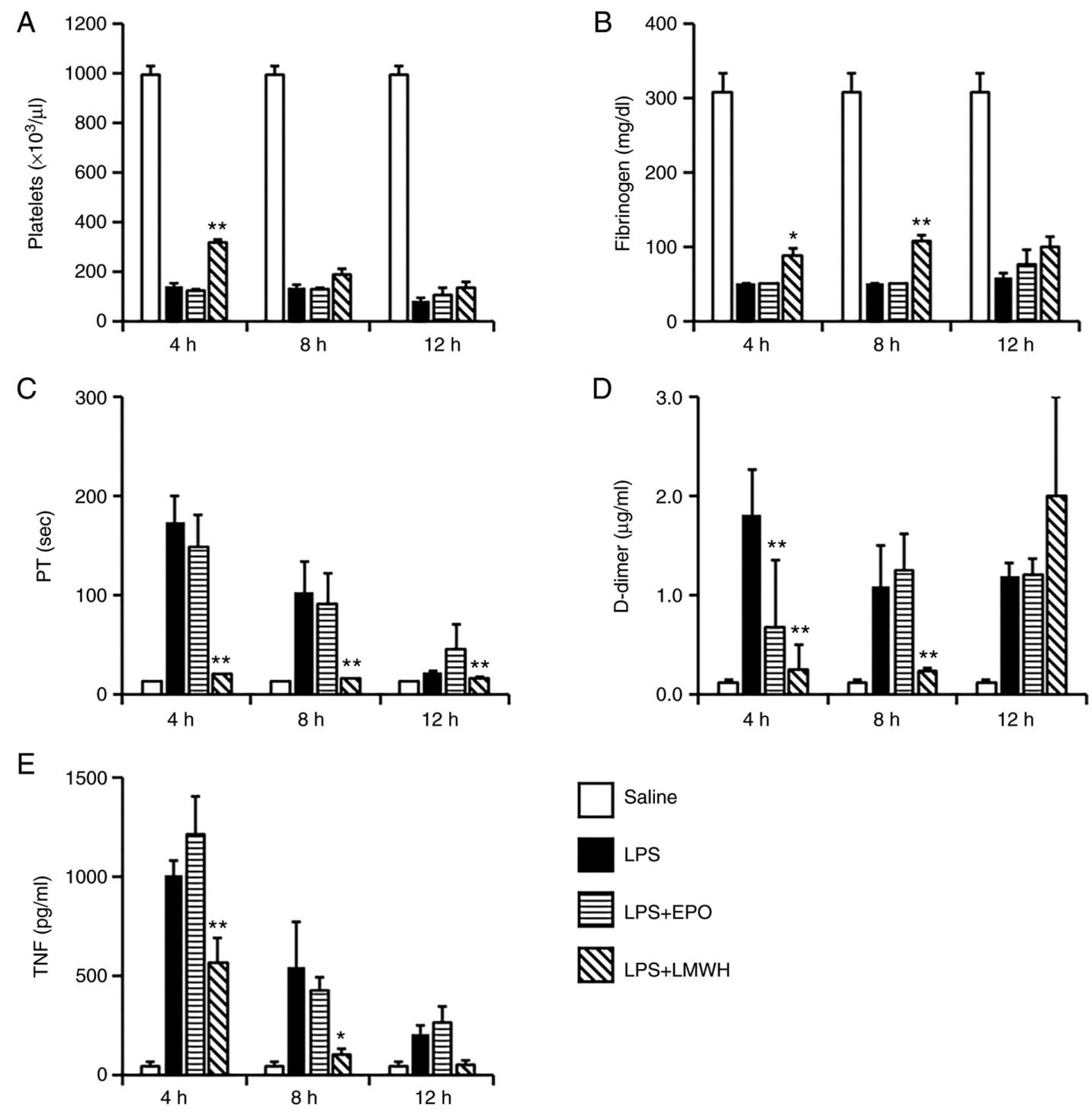

Figure 1. Changes in plasma levels of (A) platelets, (B) fibrinogen, (C) PT, (D) D-dimer and (E) TNF 4, 8 and $12 \mathrm{~h}$ after the start of LPS administration in the LPS-induced DIC model. $\mathrm{n}=7 .{ }^{*} \mathrm{P}<0.05,{ }^{* *} \mathrm{P}<0.01$ vs. LPS group. LPS, lipopolysaccharide ( $\left.5 \mathrm{mg} / \mathrm{kg} / 4 \mathrm{~h}\right) ; \mathrm{LPS}+\mathrm{EPO}, \mathrm{LPS}+$ erythropoietin (10,000 IU $\left./ \mathrm{kg} / 0.5 \mathrm{~h}\right)$; LPS+LMWH, LPS + low molecular weight heparin, (400 IU/kg/4.5 h); PT, prothrombin time; DIC, disseminated intravascular coagulation.

number of cells) x100 (\%) (25). AI was evaluated $12 \mathrm{~h}$ after LPS infusion, as no significant hepatocellular apoptosis was observed until at least $8 \mathrm{~h}$ in the pilot experiments (data not shown), although hepatocellular apoptosis was only slightly even then, and therefore evaluation of hepatocyte AI was most appropriate after $12 \mathrm{~h}$, when marked hepatocellular apoptosis was observed in rat models of LPS-induced DIC.

Statistical analysis. All data are presented as the mean \pm the standard error of the mean. Results were statistically analyzed using a Student's t-test. For multiple comparisons, P-values were adjusted using the Holm's method. $\mathrm{P}<0.05$ was considered to indicate a statistically significant difference.

\section{Results}

Changes in hemostatic parameters, TNF levels, organ function and AI in hepatocytes are shown in Figs. 1-4. No significant changes were observed in any of the parameters examined in rats treated with physiological saline and EPO or LMWH at 4,8 and $12 \mathrm{~h}$ after the start of physiological saline administration (data not shown).

Hemostatic parameters. Platelet counts fell in the LPS groups, and were unimproved in the LPS+EPO groups, while a significant increase was observed in the LPS+LMWH group at $4 \mathrm{~h}$ $(\mathrm{P}<0.01$; Fig. 1A). Plasma levels of fibrinogen decreased markedly to the point of being undetectable in the LPS and LPS+EPO groups, and this decrease was attenuated in the LPS+LMWH groups at 4 and $8 \mathrm{~h}(\mathrm{P}<0.05$ and $\mathrm{P}<0.01$, respectively; Fig. 1B). PT was prolonged in the LPS and LPS+EPO groups, and this prolongation was attenuated in the LPS+LMWH groups at all three timepoints (all $\mathrm{P}<0.01$; Fig. 1C). Significant suppression of LPS-induced D-dimer elevation was observed in the LPS+EPO group at $4 \mathrm{~h}(\mathrm{P}<0.01$; Fig. 1D), and in the LPS+LMWH groups after 4 and $8 \mathrm{~h}$ (both $\mathrm{P}<0.01$; Fig. 1D). Plasma levels of TNF were markedly increased after $4 \mathrm{~h}$ in the LPS group, followed by an early decline. The elevation of plasma TNF was not attenuated in the LPS+EPO groups, but was significantly suppressed in the LPS+LMWH groups at 4 and $8 \mathrm{~h}(\mathrm{P}<0.01$ each; Fig. 1E). 

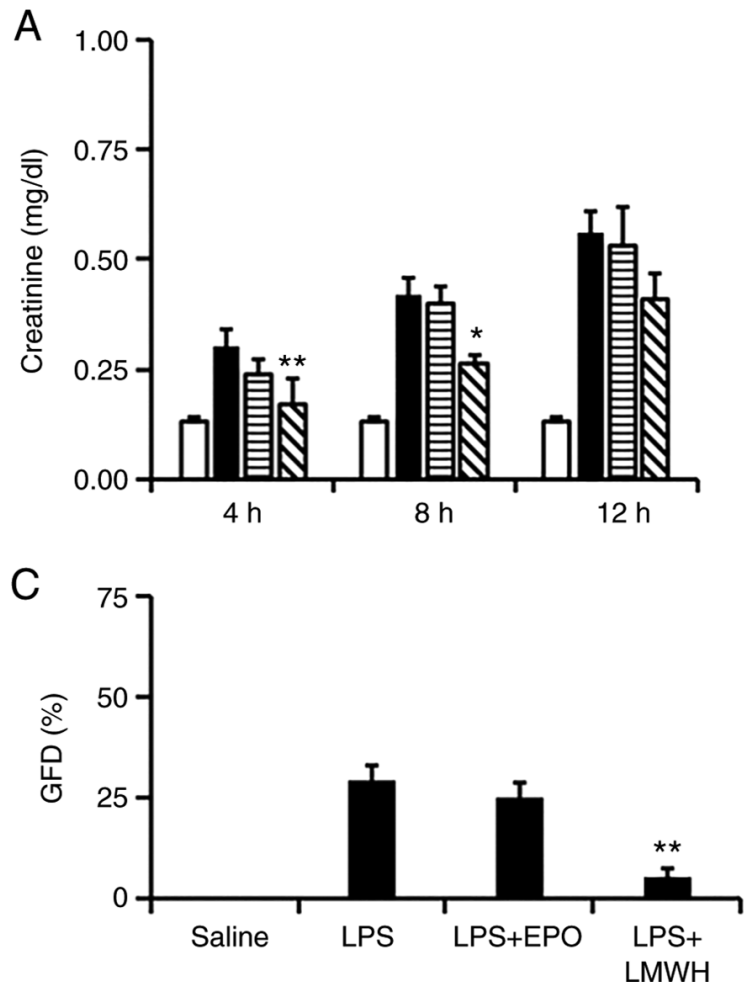
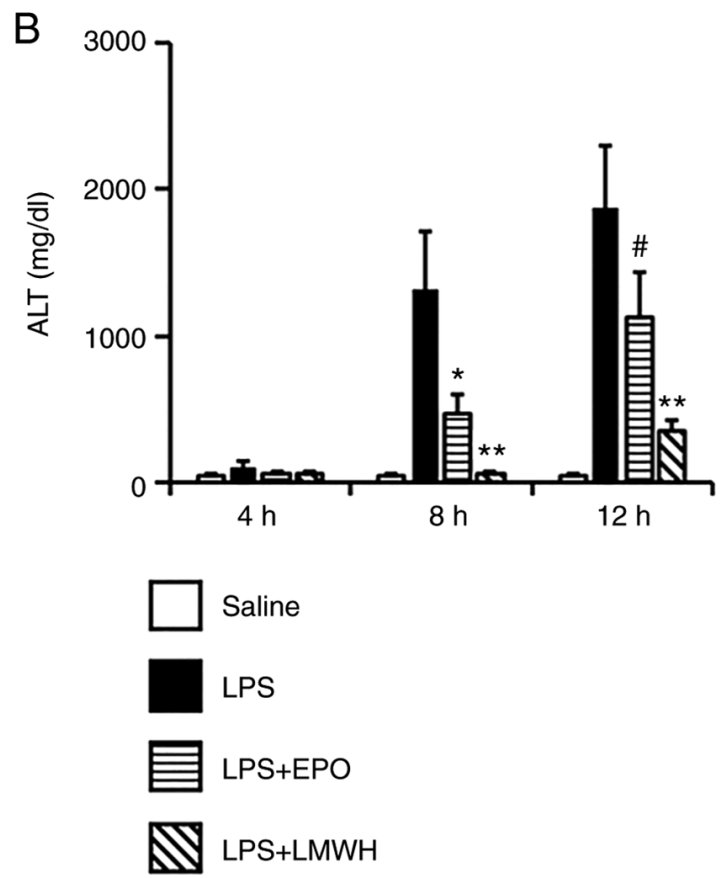

Figure 2. Changes in the plasma levels of (A) creatinine and (B) ALT 4, 8 and $12 \mathrm{~h}$ after the start of LPS administration, and the percentage of (C) glomerular fibrin deposition after $8 \mathrm{~h}$ in the LPS-induced DIC model. $\mathrm{n}=7 .{ }^{*} \mathrm{P}<0.05,{ }^{* *} \mathrm{P}<0.01$ vs. LPS group. LPS; ${ }^{\mathrm{P}}=0.062$ vs. LPS group. ALT, alanine aminotransferase; DIC, disseminated intravascular coagulation; LPS, lipopolysaccharide (5 mg/kg/4 h); LPS+EPO, LPS + erythropoietin (10,000 IU/kg/0.5 h); LPS+LMWH, LPS + low molecular weight heparin, (400 IU/kg/4.5 h).

Organ function. Plasma levels of creatinine, as an indicator of renal dysfunction, were increased during LPS infusion and increased further after infusion in the LPS groups. This elevation of plasma creatinine was not modulated in the LPS+EPO groups, but was significantly suppressed in the LPS+LMWH groups after 4 and $8 \mathrm{~h}(\mathrm{P}<0.01$ and $\mathrm{P}<0.05$, respectively; Fig. 2A). Plasma ALT levels, as an indicator of liver injury, were increased in the LPS groups after 8 and $12 \mathrm{~h}$, but were significantly suppressed in the LPS+EPO group after $8 \mathrm{~h}(\mathrm{P}<0.05$; Fig. $2 \mathrm{~B})$ and in the LPS+LMWH group after 8 and $12 \mathrm{~h}(\mathrm{P}<0.05$ and $\mathrm{P}<0.01$, respectively; Fig. $2 \mathrm{~B})$. In the LPS+EPO group, elevation of ALT levels was not significantly attenuated after $12 \mathrm{~h}$, although a suppressive tendency was still observed ( $\mathrm{P}=0.062$; Fig. 2B). GFD, presented as \% GFD, after $8 \mathrm{~h}$ is shown in Figs. 2C and 3. Significant fibrin deposition was found in the LPS groups, and was not suppressed in the LPS+EPO groups, but a significant suppression of \% GFD was observed in the LPS+LMWH groups $(\mathrm{P}<0.01$; Fig. 2C). The AI in hepatocytes is shown in Fig. 4. Marked hepatocellular apoptosis was observed in the LPS groups. In the LPS+EPO and LPS+LMWH groups, AI was significantly reduced $(\mathrm{P}<0.05$ each; Fig. 4).

\section{Discussion}

Recombinant human EPO and its analogue exert beneficial effects on organ dysfunction in several rodent models of sepsis (14-16). As severe sepsis often causes MOF due to DIC (26), the organ-protective effect of EPO apparent in animal models of sepsis has been hypothesized to be attributable,
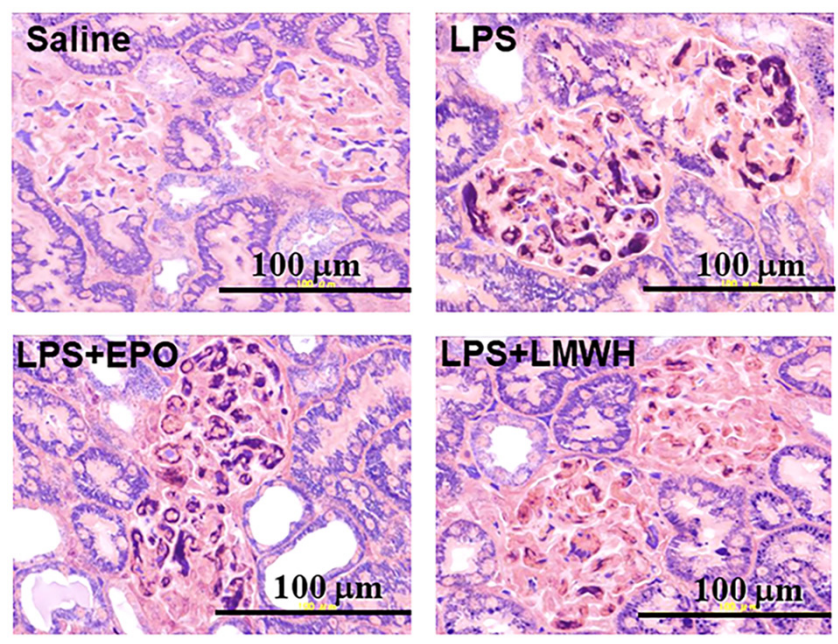

Figure 3. Effects of EPO or LMWH treatment on microthrombi formation in glomeruli $8 \mathrm{~h}$ after the start of LPS administration in the LPS-induced DIC model. Saline, physiological saline; LPS, lipopolysaccharide $(5 \mathrm{mg} / \mathrm{kg} / 4 \mathrm{~h})$; LPS+EPO, LPS + erythropoietin $(10,000 \mathrm{IU} / \mathrm{kg} / 0.5 \mathrm{~h}) ; \mathrm{LPS}+\mathrm{LMWH}$, LPS + low molecular weight heparin, (400 IU/kg/4.5 h); DIC, disseminated intravascular coagulation.

at least in part, to improvement of sepsis-induced DIC. The primary purpose of the present study was to investigate the effects of anti-apoptotic therapy on the pathophysiology of sepsis-induced DIC, using human recombinant EPO in the LPS-induced DIC model.

Reduced platelet counts and fibrinogen levels were unimproved by EPO, while elevations of plasma D-dimer levels 


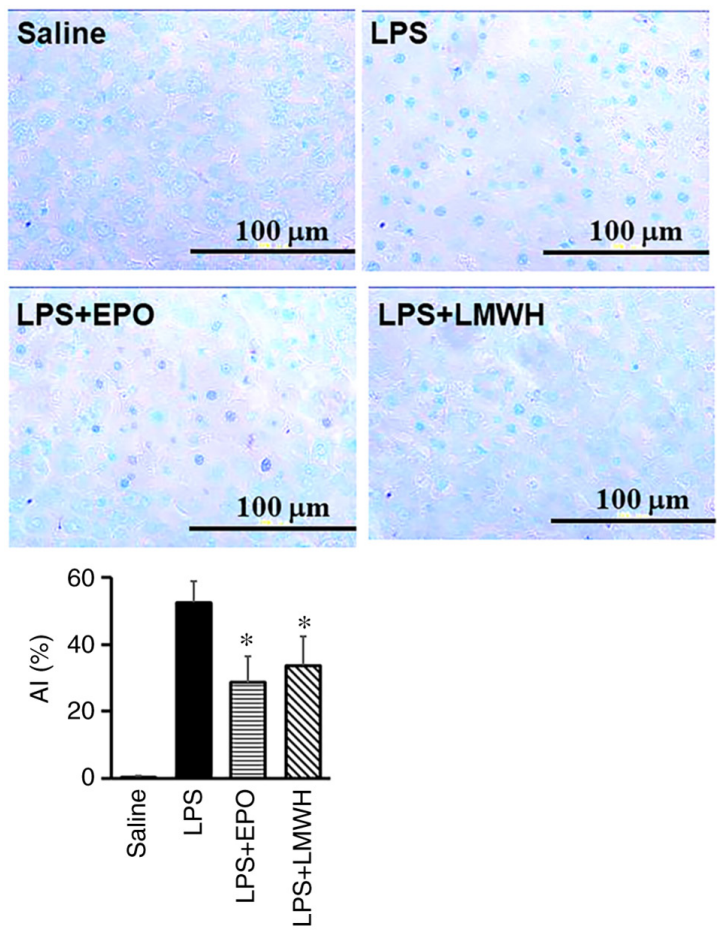

Figure 4. Effect of EPO or LMWH on hepatocyte apoptosis. Microscopy images showing apoptosis in hepatic tissue specimens $12 \mathrm{~h}$ after the start of LPS administration using TUNEL staining. The bar graph shows the change in the AI of hepatocytes after $12 \mathrm{~h}$ Results are presented as the mean \pm the standard error of the mean. $n=7$. ${ }^{*} \mathrm{P}<0.05$ vs. LPS group. AI, apoptotic index; saline, physiological saline; LPS, lipopolysaccharide $(5 \mathrm{mg} / \mathrm{kg} / 4 \mathrm{~h})$ LPS+EPO, LPS + erythropoietin $(10,000 \mathrm{IU} / \mathrm{kg} / 0.5 \mathrm{~h}) ; \mathrm{LPS}+\mathrm{LMWH}$, LPS + low molecular weight heparin, (400 IU/kg/4.5 h).

were significantly suppressed. Although EPO administration did not notably improve reduced platelet counts and fibrinogen levels, a decrease in plasma D-dimer levels may suggest that thrombus caused by LPS stimulation was improved by EPO administration. In addition, as discussed in greater detail later, the liver dysfunction developed by thrombus in the LPS model was significantly improved by EPO administration (significantly lower ALT in the LPS+EPO groups). This result also suggested that EPO treatment improved the thrombus formation that caused liver dysfunction, resulting in decreased levels of D-dimer in the LPS+EPO groups. Inflammatory cytokines, such as TNF and IL-6 are systemically upregulated, and play an important role in initiating and accelerating hemostatic activation in the rat DIC model $(27,28)$. In the present study, plasma TNF elevation remained unattenuated by EPO. The anti-inflammatory effects of EPO on sepsis-induced systemic inflammation have been examined previously, but the results have varied according to the methods of sepsis induction (15). The LPS-induced DIC model used in the present study seemed to present a severe septic pathophysiology, as the rat model of sepsis was caused by intravenous administration of high doses of LPS (5 mg/kg). Accordingly, EPO failed to exert any appreciable anti-inflammatory (anti-cytokine) effect on LPS-induced systemic inflammation in the present study.

In the LPS+LMWH groups, abnormalities in hemostatic parameters, such as platelet counts, fibrinogen levels, PT and plasma D-dimer levels were significantly improved. Elevation of plasma TNF was also notably suppressed by the administration of LMWH. In our previous studies it was reported that LMWH had several beneficial effects, such as improvement of hemostatic markers and organ dysfunction, against LPS-induced DIC $(22,23)$. The improvement of renal and hepatic dysfunction at $8 \mathrm{~h}$ was hypothesized to be due to the strong inhibition of thrombus formation by LMWH at an early stage. Since single administration of EPO did not improve LPS-induced hemostatic abnormalities, except in the D-dimer levels, modification of hemostatic abnormalities and suppression of plasma TNF elevation in the LPS+LMWH group was attributed to the anticoagulant and anti-inflammatory effects of LMWH (22).

Protective effects of EPO against sepsis-induced renal dysfunction have been reported previously, with EPO significantly attenuating renal dysfunction and ameliorating kidney histopathological changes in experimental mice (29). The difference in results between the previous and the present study may be due to the different animals used, and the severity of septic pathophysiology resulting from differences in the methods of inducing sepsis. On the other hand, hepatic dysfunction was significantly attenuated in the LPS+EPO group, as indicated by the suppression of plasma ALT elevations caused by LPS, accompanied by significant suppression of LPS-induced hepatocellular apoptosis. Although hepatoprotective effects of darbepoetin- $\alpha$, a long-acting analogue of EPO, were reported in another study using a different mouse model of septic acute liver injury (30), the results of the present study clarified that EPO was effective against hepatic dysfunction induced by high doses of LPS, further mimicking the hepatic pathophysiology of sepsis-induced DIC. In the LPS+LMWH groups, plasma ALT elevation, PT prolongation and the AI were suppressed compared with those in the LPS groups. Previously, the LMWH derivative certoparin was reported to suppress Adriamycin-induced DNA fragmentation, a significant biochemical indicator of apoptotic cell death, in heart and kidney tissues from a rat model of Adriamycin-induced oxidative cytotoxicity (31). The results of the present study suggested that LMWH may also be protective against sepsis-induced apoptosis, at least in hepatocytes, indicating a novel effect of heparinoids against LPS-induced DIC, in addition to the well-known anti-coagulant effects. From the results of the present study, a single administration of EPO was considered to improve hepatic dysfunction primarily through the exertion of anti-apoptotic effects. Administration of EPO with co-administration of LMWH may thus be more effective against LPS-induced organ dysfunctions, as both anticoagulant and anti-inflammatory effects are offered by co-administration of LMWH, in addition to the potent anti-apoptotic effects of EPO.

Several limitations should be considered when examining the results of the present study. First, TNF was the only measure of inflammatory status used. Although the results for TNF after $12 \mathrm{~h}$ in the LPS+LMWH group suggested that inflammation had disappeared, inflammatory status cannot be considered to have been accurately assessed, since other proinflammatory cytokines, including IL-1 and IL-6, were not measured. This issue thus needs to be clarified in future studies. Similarly, apoptosis requires multidimensional evaluations $(32,33)$, and evaluations using cell viability, DRAQ7 or caspase expression should be performed in the future. Finally, 
creatinine and GFD were used as markers of renal function in the present study, but measurement of urinary protein and pathological examinations may also be warranted to evaluate renal function more accurately.

The present study is the first to examine the effects of EPO on the pathophysiology of DIC, to the best of our knowledge. Although administration of EPO was ineffective against hemostatic abnormalities and systemic inflammation in LPS-induced DIC, hepatic dysfunction was significantly improved following suppression of hepatocellular apoptosis. This study also clarified that LMWH exhibited anti-apoptotic properties in LPS-induced DIC. Anti-apoptotic therapy may thus be beneficial in patients with sepsis accompanied by DIC.

\section{Acknowledgements}

Not applicable.

\section{Funding}

No funding was received.

\section{Availability of data and materials}

All data generated and/or analyzed during the present study are included in the published article.

\section{Authors' contributions}

YS and HA contributed to the experimental design, analysis of data and wrote the manuscript. FA contributed to the experiments. SY assisted with the experiments and analysis of data. EM contributed to drafting/editing of the manuscript and assisted with the analysis of data. All authors have read and approved the final manuscript. YS and HA confirm the authenticity of all the raw data.

\section{Ethics approval and consent to participate}

The present study was approved by the Committee on Animal Experimentation of Kanazawa University (Kanazawa, Japan; approval no. AP-183920).

\section{Patient consent for publication}

Not applicable.

\section{Competing interests}

The authors declare that they have no competing interests.

\section{References}

1. Levi M and Ten Cate H: Disseminated intravascular coagulation. N Engl J Med 341: 586-592, 1999.

2. Adelborg K, Larsen JB and Hvas AM: Disseminated intravascular coagulation: Epidemiology, biomarkers, and management. Br J Haematol 192: 803-818, 2021.

3. Asakura H: Classifying types of disseminated intravascular coagulation: Clinical and animal models. J Intensive Care 2: 20, 2014.

4. Angus DC and van der Poll T: Severe sepsis and septic shock. N Engl J Med 369: 840-851, 2013.
5. Brooks MB, Turk JR, Guerrero A, Narayanan PK, Nolan JP, Besteman EG, Wilson DW, Thomas RA, Fishman CE, Thompson KL, et al: Non-lethal endotoxin injection: A rat model of hypercoagulability. PLoS One 12: e0169976, 2017.

6. Schöchl H, Solomon C, Schulz A, Voelcke W, Hanke A, Van Griensven M and Redl H, Bahrami S: Thromboelastometry (TEM) findings in disseminated intravascular coagulation in a pig model of endotoxinemia. Mol Med 17: 266-272, 2011.

7. Schoergenhofer C, Schwameis M, Gelbenegger G, Buchtele N, Thaler B, Mussbacher M,Schabbauer G, Wojta J,Jilma-StohlawetzP and Jilma B: Inhibition of Protease-activated receptor (PAR1) reduces activation of the endothelium, coagulation, fibrinolysis and inflammation during human endotoxemia. Thromb Haemost 118: 1176-1184, 2018.

8. Levi M and van der Poll T: Coagulation and sepsis. Thromb Res 149: 38-44, 2017.

9. Suresh S, Rajvanshi PK and Noguchi CT: The Many Facets of erythropoietin physiologic and metabolic response. Front Physiol 10: 1534, 2019.

10. Bonomini M, Del Vecchio L, Sirolli V and Locatelli F: New Treatment approaches for the anemia of CKD. Am J Kidney Dis 67: 133-142, 2016.

11. Singh AK, Szczech L, Tang KL, Barnhart H, Sapp S, Wolfson M and Reddan D; CHOIR Investigators: Correction of anemia with epoetin alfa in chronic kidney disease. N Engl J Med 355: 2085-2098, 2006.

12. Yang J, Zhou J, Wang X, Wang X, Ji L, Wang S, Chen X and Yang L: Erythropoietin attenuates experimental contrast-induced nephrology: A role for the janus Kinase 2/Signal transducer and activator of transcription 3 signaling pathway. Front Med (Lausanne) 8: 634882, 2021.

13. Golmohammadi MG, Banaei S, Nejati K and Chinifroush-Asl MM: Vitamin D3 and erythropoietin protect against renal ischemia-reperfusion injury via heat shock protein 70 and microRNA-21 expression. Sci Rep 10: 20906, 2020.

14. Thiemermann C: Beneficial effects of erythropoietin in preclinical models of shock and organ failure. Crit Care 11: 132, 2007.

15. Silva I, Alipio C, Pinto R and Mateus V: Potential anti-inflammatory effect of erythropoietin in non-clinical studies in vivo: A systematic review. Biomed Pharmacother 139: 111558, 2021.

16. Li K, Liu TX, Li JF, Ma YR, Liu ML, Wang YQ, Wu R, Li B, Shi LZ and Chen C: rhEPO inhibited cell apoptosis to alleviate acute kidney injury in sepsis by AMPK/SIRT1 activated autophagy. Biochem Biophys Res Commun 517: 557-565, 2019.

17. Tu Q, Zhu Y, Yuan Y, Guo L, Liu L, Yao L, Zou Y, Li J and Chen F: Gypenosides inhibit inflammatory response and apoptosis of endothelial and epithelial cells in LPS-Induced ALI: A study based on bioinformatic analysis and in vivo/vitro experiments. Drug Des Devel Ther 15: 289-303, 2021.

18. Duan H, Zhang Q, Liu J, Li R, Wang D, Peng W and Wu C: Suppression of apoptosis in vascular endothelial cell, the promising way for natural medicines to treat atherosclerosis. Pharmacol Res 168: 105599, 2021.

19. Bombeli T, Karsan A, Tait JF and Harlan JM: Apoptotic vascular endothelial cells become procoagulant. Blood 89: 2429-2442, 1997.

20. Toltl LJ, Swystun LL, Pepler L and Liaw PC: Protective effects of activated protein C in sepsis. Thromb Haemost 100: 582-592, 2008.

21. Woo S, Krzyzanski W and Jusko WJ: Pharmacokinetic and pharmacodynamic modeling of recombinant human erythropoietin after intravenous and subcutaneous administration in rats. J Pharmacol Exp Ther 319: 1297-1306, 2006.

22. Asakura H, Sano Y, Yoshida T, Omote M, Ontachi Y, Mizutani T, Yamazaki M, Morishita E, Takami A, Miyamoto K and Nakao S: Beneficial effect of low-molecular-weight heparin against lipopolysaccharide-induced disseminated intravascular coagulation in rats is abolished by coadministration of tranexamic acid. Intensive Care Med 30: 1950-1955, 2004.

23. Asakura H, Sano Y, Omote M, Yoshida T, Ontachi Y, Mizutani T, Kaneda M, Yamazaki M, Morishita E, Takami A, et al: Significance of decreased plasma D-dimer levels following lipopolysaccharide-induced disseminated intravascular coagulation in rats. Int J Hematol 79: 394-399, 2004.

24. Koroglu TF, Yilmaz O, Ozer E, Baskin H, Gokmen N, Kumral A, Duman M and Ozkan H: Erythropoietin attenuates lipopolysaccharide-induced splenic and thymic apoptosis in rats. Physiol Res 55: 309-316, 2006.

25. Zhou HB, Chen JM, Cai JT, Du Q and Wu CN: Anticancer activity of genistein on implanted tumor of human SG7901 cells in nude mice. World J Gastroenterol 14: 627-631, 2008. 
26. Colantuoni A, Martini R, Caprari P, Ballestri M, Capecchi PL, Gnasso A, Lo Presti R, Marcoccia A, Rossi M and Caimi G: COVID-19 Sepsis and microcirculation dysfunction. Front Physiol 11: 747, 2020.

27. Okajima K: Regulation of inflammatory responses by natural anticoagulants. Immunol Rev 184: 258-274, 2001.

28. Suga Y, Kubo A, Katsura H, Staub Y, Tashiro K, Yamada S, Morishita E and Asakura H: Detailed exploration of pathophysiology involving inflammatory status and bleeding symptoms between lipopolysaccharide- and tissue factor-induced disseminated intravascular coagulation in rats. Int $\mathrm{J}$ Hematol 114: $172-178,2021$

29. Stoyanoff TR, Todaro JS, Aguirre MV, Zimmermann MC and Brandan NC: Amelioration of lipopolysaccharide-induced acute kidney injury by erythropoietin: Involvement of mitochondria-regulated apoptosis. Toxicology 318: 13-21, 2014.

30. Le Minh K, Klemm K, Abshagen K, Eipel C, Menger MD and Vollmar B: Attenuation of inflammation and apoptosis by preand posttreatment of darbepoetin-alpha in acute liver failure of mice. Am J Pathol 170: 1954-1963, 2007.
31. Deepa PR and Varalakshmi P: Influence of a low-molecular-weight heparin derivative on the nitric oxide levels and apoptotic DNA damage in adriamycin-induced cardiac and renal toxicity. Toxicology 217: 176-183, 2006.

32. Zhang W, Tian Y, Gao Q, Li X, Li Y, Zhang J, Yao C, Wang Y, Wang $\mathrm{H}$, Zhao $\mathrm{Y}$, et al: Inhibition of apoptosis reduces diploidization of haploid mouse embryonic stem cells during differentiation. Stem Cell Reports 15: 185-197, 2020.

33. Wang H, Zhang W, Gao Q, Cao X, Li Y, Li X, Min Z, Yu Y, Guo Y and Shuai L: Extractive from Hypericum ascyron L promotes serotonergic neuronal differentiation in vitro. Stem Cell Res 31: 42-50, 2018.

(7) $\Theta$ This work is licensed under a Creative Commons BY NC ND Attribution-NonCommercial-NoDerivatives 4.0 International (CC BY-NC-ND 4.0) License. 